This item was submitted to Loughborough's Research Repository by the author.

Items in Figshare are protected by copyright, with all rights reserved, unless otherwise indicated.

\title{
Nernst effect in poor conductors and the cuprate superconductors
}

PLEASE CITE THE PUBLISHED VERSION

LICENCE

CC BY-NC-ND 4.0

\section{REPOSITORY RECORD}

Alexandrov, A.S., and V.N. Zavaritsky. 2019. "Nernst Effect in Poor Conductors and the Cuprate Superconductors". figshare. https://hdl.handle.net/2134/1168. 


\title{
Nernst effect in poor conductors and in the cuprate superconductors
}

\author{
A. S. Alexandrov and V. N. Zavaritsky \\ Department of Physics, Loughborough University, Loughborough LE11 3TU, United Kingdom
}

\begin{abstract}
We calculate the Nernst signal in disordered conductors with the chemical potential near the mobility edge. The Nernst effect originates from interference of itinerant and localised-carrier contributions to the thermomagnetic transport. It reveals a strong temperature and magnetic field dependence, which describes quantitatively the anomalous Nernst signal in high- $T_{c}$ cuprates.
\end{abstract}

PACS numbers: 74.40.+k, 72.15.Jf, 74.72.-h, 74.25.Fy

Thermomagnetic effects appear in conductors subjected to a longitudinal temperature gradient $\nabla T$ (in $x$ direction) and a perpendicular magnetic field $\mathbf{B}$ in $z$ direction. The transverse Nernst-Ettingshausen effect (further the Nernst effect) is the appearance of a transverse electric field $E_{y}$ in the third direction. This effect as well as the longitudinal one were discovered by Nernst and Ettingshausen in a bismuth plate in 1886 [1]. The effect is known to be small in ordinary metals. Indeed in the framework of a single-band effective mass approximation it appears only in the second order with respect to the degeneracy $k_{B} T / E_{F} \ll 1$ due to a so-called Sondheimer cancellation [2], if the relaxation time $\tau(E)$ depends on energy. If $\tau$ does not depend on energy, the Nernst signal disappears even for nondegenerate carriers in the same approximation [3].

Sufficiently large positive Nernst effect was found in high- $T_{c}$ cuprates in the vicinity of the resistive transition temperature $T_{c}$ [4]. As in conventional superconductors it was attributed to motion of vortices down the thermal gradient while a small negative signal, measured well above $T_{c}[\underline{5}$, was ascribed to the relaxation time decreasing with carrier energy. Such a negative signal may also originate from the counterflow of carriers with opposite sign (the familiar ambipolar Nernst effect), as explained by a simple two band model for electrons and holes with different mobilities [6], and/or from a charged density wave order [7], as observed in $\mathrm{NbSe}_{2}$.

Recently much attention has been paid to the anomalously enhanced positive Nernst signal observed well above $\mathrm{T}_{c}$ in $\mathrm{La}_{2-x} \mathrm{Sr}_{x} \mathrm{CuO}_{4}$ (LSCO-x) in a wide range of doping $x[8]$. It has been attributed to the vortex motion, since the Sondheimer cancellation renders any 'normal state' scenario allegedly implausible [8]. As a result, the magnetic phase diagram of the cuprates has been revised with the upper critical field $H_{c 2}(T)$ curve not ending at $T_{c}$ but at a much higher temperature [9, 10]. Most surprisingly, Refs. [9, 10] estimated $H_{c 2}$ at the superconducting transition temperature, $T_{c}$, as high as 40-150 Tesla. Wang et al. 9] argued that the large Nernst signal supports a scenario 11], where the superconducting order parameter (i.e. the Bogoliubov- Gor'kov anomalous average $F\left(\mathbf{r}, \mathbf{r}^{\prime}\right)=\left\langle\psi_{\downarrow}(\mathbf{r}) \psi_{\uparrow}\left(\mathbf{r}^{\prime}\right\rangle\right)$ does not disappear at $T_{c}$ but at much higher (pseudogap) temperature $T^{*}$. Several other works 12] have also suggested that the anomalous Nernst effect is a result of the fluctuations of the super- conducting order parameter above $T_{c}$.

However, any phase fluctuation scenario is difficult to reconcile with the extremely sharp resistive and magnetic transitions at $T_{c}$ in single crystals of cuprates. The uniform magnetic susceptibility at $T>T_{c}$ is paramagnetic, and the resistivity is perfectly 'normal' showing only a few percent positive or negative magnetoresistance. Both in-plane 13, 14, 15, 16] and out-of-plane 17] resistive transitions remain sharp in the magnetic field in high quality samples providing a reliable determination of the genuine $H_{c 2}(T)$. The vortex entropy estimated from the Nernst signal was found an order of magnitude smaller than the difference between the entropy of the superconducting state and the extrapolated entropy of the normal state obtained by specific heat measurements [18]. These and some other observations [19] do not support any superconducting order parameter above $T_{c}$.

In this Letter we calculate the Nernst signal for disordered conductors with the chemical potential, $\mu$, close to the mobility edge. No 'Sondheimer cancellation' of the signal exists in this case. Mott's law 20] for the variablerange-hopping conduction of carriers localised below the mobility edge together with the Boltzmann kinetics for itinerant fermionic carriers or preformed bosonic pairs above the edge yields the Nernst signal, which agrees quantitatively with the signal in the superconducting cuprates at temperatures, $T>T_{c}(B)$ above the resistive phase transition.

The Nernst voltage is expressed in terms of the kinetic coefficients $\sigma_{i j}$ and $\alpha_{i j}$ as $[3$

$$
e_{y}(T, B) \equiv-\frac{E_{y}}{\nabla_{x} T}=\frac{\sigma_{x x} \alpha_{y x}-\sigma_{y x} \alpha_{x x}}{\sigma_{x x}^{2}+\sigma_{x y}^{2}},
$$

where the current density per spin is given by $j_{i}=$ $\sigma_{i j} E_{j}+\alpha_{i j} \nabla_{j} T$. Carriers in doped semiconductors and disordered metals occupy states localised by disorder and itinerant Bloch-like states. Both types of carriers contribute to the transport properties, if the chemical potential $\mu$ (or the Fermi level) is close to the energy, where the lowest itinerant state appears (i.e. to the mobility edge). Superconducting cuprates are among such poor conductors and their superconductivity appears as a result of doping, which inevitably creates disorder. Differently from 3D-conductors, the localised states cannot be 'screened' off by the itinerant carriers in these almost two-dimensional conductors even at high density of car- 
riers. It is well known that in two dimensions a bound state exists for any attraction, however weak. Indeed, there is strong experimental evidence for the coexistence of itinerant and localised carriers in cuprates in a wide range of doping [21].

The standard Boltzmann equation in the relaxation time approximation yields for itinerant carriers

$$
\begin{gathered}
\sigma_{x x}=-e^{2} \sum_{\mathbf{k}} v_{x}^{2} \tilde{\tau}\left(E_{\mathbf{k}}\right) \frac{\partial f\left(E_{\mathbf{k}}\right)}{\partial E_{\mathbf{k}}} \\
\sigma_{y x}=-e^{3} B \sum_{\mathbf{k}} \frac{v_{x}^{2}}{m_{y}} \tau\left(E_{\mathbf{k}}\right) \tilde{\tau}\left(E_{\mathbf{k}}\right) \frac{\partial f\left(E_{\mathbf{k}}\right)}{\partial E_{\mathbf{k}}} \\
\alpha_{x x}=-e \sum_{\mathbf{k}} \frac{E_{\mathbf{k}}-\mu}{T} v_{x}^{2} \tilde{\tau}\left(E_{\mathbf{k}}\right) \frac{\partial f\left(E_{\mathbf{k}}\right)}{\partial E_{\mathbf{k}}} \\
\alpha_{y x}=-e^{2} B \sum_{\mathbf{k}} \frac{E_{\mathbf{k}}-\mu}{T} \frac{v_{x}^{2}}{m_{y}} \tau\left(E_{\mathbf{k}}\right) \tilde{\tau}\left(E_{\mathbf{k}}\right) \frac{\partial f\left(E_{\mathbf{k}}\right)}{\partial E_{\mathbf{k}}}
\end{gathered}
$$

where $\mathbf{v}=\nabla_{\mathbf{k}} E_{\mathbf{k}}$ is the group velocity, $E_{\mathbf{k}}$ is the band dispersion, $1 / m_{i}=\partial^{2} E_{\mathbf{k}} / \partial k_{i}^{2}$ is the inverse mass tensor, which is assumed to be diagonal, $\hbar=c=1, f\left(E_{\mathbf{k}}\right)$ is the equilibrium distribution function, and

$$
\tilde{\tau}\left(E_{\mathbf{k}}\right)=\frac{\tau\left(E_{\mathbf{k}}\right)}{1+\left[e \tau\left(E_{\mathbf{k}}\right) B\right]^{2} /\left(m_{x} m_{y}\right)} .
$$

Both $\alpha_{x x}$ and $\alpha_{x y}$ vanish at $T=0$ for degenerate fermions with any $\tau\left(E_{\mathbf{k}}\right)$, if their band is parabolic, so that $1 / m_{i}$ does not depend on $\mathbf{k}$. When $\tau$ does not depend on energy two terms in the numerator of $e_{y}$, Eq.(1) cancel each other at any temperature in the parabolic approximation. However, a generalization of this Sondheimer cancellation for any band dispersion is flawed (see, also Ref. 7, 22]). The most striking example is a halffilled band. Modelling this band by the familiar tightbinding dispersion, $E_{\mathbf{k}}=-2 t\left[\cos \left(k_{x}\right)+\cos \left(k_{y}\right)\right]$ yields $1 / m_{x, y}=\cos \left(k_{x, y}\right) / m$, where $m=1 /(2 t), t$ is the nearestneighbour hopping integral, and $\mu=0$ for the half-filling (we take the lattice constant $a=1$ ). Then by parity, $\sigma_{y x}=\alpha_{x x}=0$, but $\alpha_{y x}$ is very large. Indeed calculating integrals, Eq.(2) and Eq.(5) we obtain at $k_{B} T \ll t$

$$
e_{y}=-\frac{2 t}{e T \Theta}\left(1-2 \Theta \ln ^{-1} \frac{1+\Theta}{|1-\Theta|}\right) \text {, }
$$

where $\Theta=e B \tau / m$. The Nernst signal is negative and super-linear, $e_{y} \approx-(2 t / 3 e T)\left(\Theta+4 \Theta^{3} / 15\right)$ at small $\Theta \ll 1$ with the minimum at $\Theta=1$. It changes sign in a strong field, $\Theta>1$, as shown in Fig.1 inset. In this simple example the number of electrons in the lower half of the band is equal to the number of holes in the upper half. As a result we arrive to a substantial negative Nernst voltage, Eq.(7), while both, the Hall effect, $R_{H}=-B^{-1} \sigma_{y x} /\left(\sigma_{x x}^{2}+\sigma_{y x}^{2}\right)$ and the thermopower,

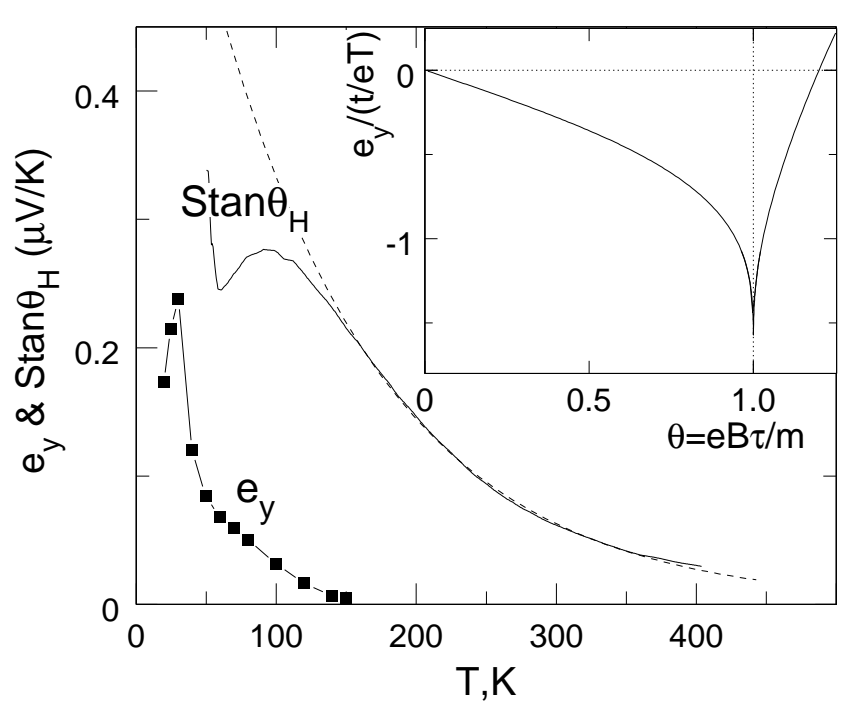

FIG. 1: The Nernst signal, $e_{y}$, and $S \tan \Theta_{H}$ in $Y B a_{2} C u_{3} O_{6.4}$ at $\mathrm{B}=1$ Tesla [10]. Inset: $e_{y}(B)$ in the half-filled band, Eq.(7).

$S=-\alpha_{x x} / \sigma_{x x}$, equal to zero at any temperature. Hence, the Sondheimer cancellation is an exception, rather than a rule. However, the thermomagnetic transport in the half-filled band, Fig.1 inset, does not describe the experimental results in cuprates. In particular, Eq.(7) yields a wrong sign of $e_{y} \approx-60 \mu \mathrm{V} / \mathrm{K}$ and the magnitude, which is at least one order larger than observed with the typical values of $\Theta=10^{-2}$ and $k_{B} T / t=10^{-2}[4,8,[9,10,18]$. Moreover, in disagreement with the half-filled band result, the Sondheimer cancellation, $S \tan \Theta_{H} \gg e_{y}$, holds in a wide temperature range, as shown in Fig.1 for $Y \mathrm{Ba}_{2} \mathrm{Cu}_{3} \mathrm{O}_{6.4}$. Here $S \tan \Theta_{H}=\sigma_{y x} \alpha_{x x} /\left(\sigma_{x x}^{2}+\sigma_{x y}^{2}\right)$ represents the second term in Eq.(1); $\mathrm{S}$ and the Hall angle, $\Theta_{H} \approx \tan \Theta_{H}=B R_{H} / \rho$, were measured independently. As it is clearly seen from Fig.1, $e_{y}$ and $S \tan \Theta_{H}$ are of the same order at sufficiently low temperatures, also in disagreement with the half-filled band results. Very similar trends of $e_{y}$ and $S \tan \Theta_{H}$ were obtained for overdoped LSCO-02 using independent measurements of $S, \rho$, and $R_{H}$ [23], in particular, $e_{y}$ and $S \tan \Theta_{H}$ are of the same order near $T_{c}$.

To account for these findings more realistic model is required. When the chemical potential is near the mobility edge, the effective mass approximation can be applied. In this case, there is no Nernst signal from itinerant carriers alone, if $\tau$ is a constant. However, now the localised carriers contribute to the longitudinal transport, so that $\sigma_{x x}$ and $\alpha_{x x}$ in Eq.(1) should be replaced by $\sigma_{x x}+\sigma_{l}$ and $\alpha_{x x}+\alpha_{l}$, respectively. Since the Hall mobility of localised carriers is often much smaller than their drift mobility [20], there is no need to add their contributions to the transverse kinetic coefficients. Neglecting the orbital effects $(\Theta \ll 1[8,9,[10])$ one obtains

$$
e_{y}(T, B)=\frac{\sigma_{l} \alpha_{y x}-\sigma_{y x} \alpha_{l}}{\left(\sigma_{x x}+\sigma_{l}\right)^{2}}
$$


When the chemical potential lies near the bottom of the band $(\mu \approx-4 t), \alpha_{y x}$, Eq.(5), and $\sigma_{y x}$, Eq.(3), are positive, but the thermopower of localised electrons with the energy below $\mu$ is negative, $\alpha_{l}<0$. Hence, there is no further 'cancellation' in the numerator of Eq.(8) in this electron-doping regime. When the chemical potential is near the top of the band $(\mu \approx 4 t), \alpha_{y x}$ remains positive, but $\sigma_{y x}$ is negative and $\alpha_{l}$ is positive, so that there is no cancellation in the hole-doping regime either. In the superconducting cuprates the conductivity of itinerant carriers $\sigma_{x x}$ dominates over the conductivity $\sigma_{l}$ of localised carriers [21], $\sigma_{x x} \gg \sigma_{l}$, which allows us to simplify Eq.(8) as

$$
\frac{e_{y}}{\rho}=\frac{k_{B}}{e} r \theta \sigma_{l}
$$

where $\rho=1 /\left[(2 s+1) \sigma_{x x}\right]$ is the resistivity, $s$ is the carrier spin, and $r$ is a constant,

$$
\frac{r}{2 s+1}=\left(\frac{e\left|\alpha_{l}\right|}{k_{B} \sigma_{l}}+\frac{\int_{0}^{\infty} d E E(E-\mu) \partial f(E) / \partial E}{k_{B} T \int_{0}^{\infty} d E E \partial f(E) / \partial E}\right)
$$

Here $N(E)$ is the density of states (DOS) near the band edge $(E=0)$, and $\mu$ is now taken with respect to the edge. The ratio $e\left|\alpha_{l}\right| / k_{B} \sigma_{l}$ is a number of the order of one. For example, $e\left|\alpha_{l}\right| / k_{B} \sigma_{l} \approx 2.4$, if $\mu=0$ and the conductivity index $\nu=1$ [24]. Calculating the integrals in Eq.(10) yields $r \approx 14.3$ for fermions $(s=1 / 2)$, and $r \approx 2.4$ for bosons $(s=0)$.

The Nernst signal, Eq.(9), is positive, and its maximum value $e_{y}^{\max } \approx\left(k_{B} / e\right) r \Theta$ is about $5-10 \mu \mathrm{V} / \mathrm{K}$ with $\Theta=10^{-2}$ and $\sigma_{l} \approx \sigma_{x x}$, as observed 8, 18]. Actually, the magnetic and temperature dependencies of the unusual Nernst effect in the overdoped LSCO are quantitatively described by Eq.(9), if $\sigma_{l}$ obeys the Mott's law,

$$
\sigma_{l}=\sigma_{0} \exp \left[-\left(T_{0} / T\right)^{x}\right]
$$

where $\sigma_{0}$ is about a constant. The exponent $x$ depends on the type of localised wavefunctions and variation of DOS, $N_{l}$ below the mobility edge [20, 25, 26]. In two dimensions one has $x=1 / 3$ and $T_{0} \approx 8 \alpha^{2} /\left(k_{B} N_{l}\right)$, where $N_{l}$ is at the Fermi level [27].

If the magnetic field is strong enough [28], the radius of the 'impurity' wave function $\alpha^{-1}$ is about the magnetic length, $\alpha \approx \sqrt{e B}$. If the relaxation time of itinerant carriers is due to the particle-particle collisions, the Hall angle depends on temperature as $\Theta \propto T^{-2}$, and the resistivity is linear, $\rho \propto T$ since the density of itinerant carriers is linear in temperature, both for fermionic [29] or bosonic (e.g. bipolaronic) carriers [30]. Hence, the model explains the temperature dependence of the normal-state Hall angle and resistivity in cuprates at high temperatures. Finally, using Eq.(9) and Eq.(11) the Nernst signal is given by

$$
\frac{e_{y}}{B \rho}=a(T) \exp \left[-b(B / T)^{1 / 3}\right]
$$

where $a(T) \propto T^{-2}$ and $b=2\left[e /\left(k_{B} N_{l}\right)\right]^{1 / 3}$ is a constant. Evidently, the phonon drag effect should be taken

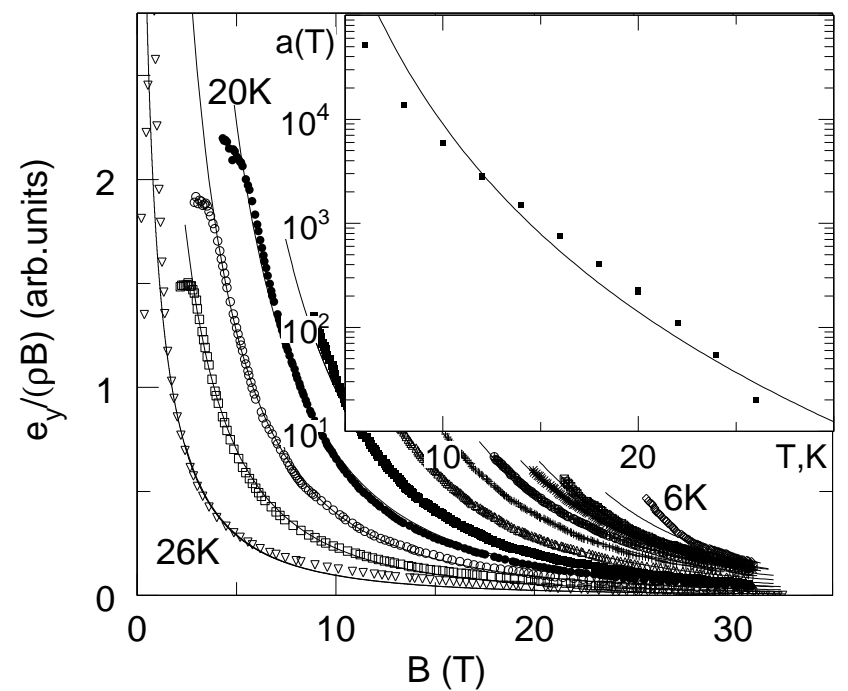

FIG. 2: Eq.(12) fits the experimental signal (symbols) in LSCO-02 [8] with $b=7.32(\mathrm{~K} / \text { Tesla })^{1 / 3}$. Inset shows $a(T)$ obtained from the fit (dots) together with $a \propto T^{-6}$ (line).

into account at sufficiently low temperatures in any realistic model. One can account for this effect by replacing $E_{\mathbf{k}}$ in Eq.(4) and Eq.(5) by $E_{\mathbf{k}}+m v_{s}^{2} \tau_{p h} / \tau_{e-p h}$ 3]. Here $v_{s}$ is the sound velocity, $\tau_{p h} \propto T^{-4}$ is the phonon relaxation time due to the phonon-phonon scattering, and $\tau_{e-p h}$ is the electron (hole) relaxation time caused by electron-phonon collisions. In two dimensions $\tau_{e-p h} \propto T^{-1}$ [31], so that $a(T)$ in Eq.(12) is enhanced by the drag effect as $a(T) \propto T^{-6}$. The theoretical field dependence of $e_{y} /(B \rho)$, Eq.(12), is in excellent quantitative agreement with the experiment, as shown in Fig.2 for $b=7.32(\mathrm{~K} / \text { Tesla })^{1 / 3}$. The corresponding temperature dependence of $a(T)$ follows closely $T^{-6}$, inset to Fig.2. The density of impurity states $N_{l}=8 e /\left(b^{3} k_{B}\right)$ is about $0.4 \times 10^{14} \mathrm{~cm}^{-2}(\mathrm{eV})^{-1}$, which corresponds to the number of impurities $N_{i m} \lesssim 10^{21} \mathrm{~cm}^{-3}$, as it should be.

In agreement with the experiment [8, 9, 10], our model of thermal magnetotransport predicts anomalous Nernst signal in cuprates only within the doping interval, where superconductivity is observed. Since the chemical potential is well below the mobility edge in the nonsuperconducting underdoped cuprates [21], and it is deep inside the Bloch band in heavily doped samples, there is no 'interference' of itinerant and localised-carrier contributions in these extreme regimes. If carriers are fermions, then $S \tan \Theta_{H}$ should be larger or of the same order as $e_{y}$, because their ratio is proportional to $\sigma_{x x} / \sigma_{l} \gg 1$ in our model. Although it is the case in many cuprates (eg., Fig.1, and the text below), a noticeable suppression of $S \tan \Theta_{H}$, as compared with $e_{y}$, was reported to occur close to $T_{c}$ in strongly underdoped LSCO and in a number of Bi2201 crystals [8]. These observations could be generally understood if we take into account that un- 
derdoped cuprates are strongly correlated systems, so that a substantial part of carriers is (most probably) preformed bosonic pairs 32]. The second term in Eq.(10) vanishes for (quasi)two dimensional itinerant bosons, because the denominator diverges logarithmically. Hence, their contribution to the thermopower is logarithmically suppressed. It can be almost cancelled by the opposite sign contribution of the localised carriers, even if $\sigma_{x x} \gg \sigma_{l}$. When it happens, the Nernst signal is given by $e_{y}=\rho \alpha_{x y}$, where $\alpha_{x y} \propto \tau^{2}$, Eq.(5). Differently from that of fermions, the relaxation time of bosons is enhanced critically near the Bose-Einstein condensation temperature, $T_{c}(B), \tau \propto\left[T-T_{c}(B)\right]^{-1 / 2}$, as in atomic Bose-gases [33]. Providing $S \tan \Theta_{H} \ll e_{y}$, this critical enhancement of the relaxation time describes well the temperature dependence of $e_{y}$ in Bi2201 and in strongly underdoped LSCO close to $T_{c}(B)$. If some segments of a large Fermisurface survive in underdoped cuprates, the Bose liquid of preformed pairs coexists with the fermionic carriers. The degenerate fermions virtually do not contribute to the thermal transport, but they dominate the longitudinal and transverse electric transport. Hence, the Hall coefficient and resistivity data could not present a behavior correlated with that of the Nernst signal.

In conclusion, we calculated the Nernst signal in disordered conductors with the chemical potential near the mobility edge, and found no 'Sondheimer cancellation' of the signal. 'Sondheimer cancellation' is also absent in the half-filled band, where the Hall effect and the thermopower are zero, but the Nernst signal is large and negative. In contrast with the half-filled band, the model with itinerant and localised fermions and/or charged bosons describe quantitatively the anomalous Nernst effect in high- $T_{c}$ cuprates as a normal state phenomenon above the resistive phase transition. Our results strongly support any microscopic theory of cuprates, which describes the state above the resistive and magnetic phase transition as perfectly 'normal', $F\left(\mathbf{r}, \mathbf{r}^{\prime}\right)=0$. Differently from 9,10 the present model does not require a radical revision of the magnetic phase diagram of cuprates [34].

This work was supported by the Leverhulme Trust (grant F/00261/H). We would like to thank W. Y. Liang and K. K. Lee for helpful discussions.
[1] A. Ettingshausen, W. Nernst, Wied. Ann. 29,343 (1886).

[2] E.H. Sondheimer, Proc. Roy. Soc. 193, 484 (1948).

[3] A. Anselm, Introduction of Semiconductor Theory (Prentice and Hall, New Jersey, 1981); I.M. Tsidil'kovskii, Thermomagnetic effects in semiconductors (Infosearch Limited, London, 1962).

[4] T.T.M. Palstra, et al., Phys. Rev. Lett. 64, 3090 (1990); M.Zeh, et al., Phys. Rev. Lett. 64, 3195 (1990); M. Galffy, A. Freimuth, and U. Murek, Phys. Rev. B 41, 11029 (1990); S.J. Hagen, et al., Phys. Rev. B 42, 6777 (1990); N.V. Zavaritsky, A.V. Samoilov, A.A. Yurgens, Physica C180, 417 (1991); A. Dascoulidou, et al., Physica C201, 202 (1992); M. Oussena, et al., Phys. Rev. B46, 528 (1992).

[5] J.A. Clayhold, et al., Phys. Rev. B50, 4252 (1994).

[6] S. Lambrecht, M. Ausloos, Phys. Rev. B 53, 14047(1996).

[7] R. Bel, K. Behnia, H. Berger, Phys. Rev. Lett. 91, 066602 (2003); V. Oganesyan, I. Ussishkin, cond-mat/0312588

[8] Z.A. Xu, et al., S. Uchida, Nature (London) 406, 486 (2000); Y. Wang, et al., Phys. Rev. B 64, 224519 (2001).

[9] Y. Wang, N.P.et al., Phys. Rev. Lett. 88, 257003 (2002); Y. Wang, et al., Science 299, 86 (2003).

[10] N.P. Ong, et al., cond-mat/0312213 ibid/0110215.

[11] V.J. Emery, S.A. Kivelson, Nature, 374, 434 (1995).

[12] I. Ussishkin, S.L. Sondhi, D.A. Huse, Phys. Rev. Lett. 89, 287001 (2002); S. Tan, K. Levin, cond-mat/0302248 S. Mukerjee, D.A. Huse, ibid /0307005; C. Honerkamp, P.A. Lee, ibid /0309469.

[13] A.P. Mackenzie, et al., Phys. Rev. Lett. 71, 1238 (1993)

[14] M.S. Osofsky, et al., Phys. Rev. Lett. 71, 2315 (1993); ibid 72, 3292 (1994).

[15] D.D. Lawrie, et al.,Low Temp. Phys. 107, 491 (1997)

[16] V.F. Gantmakher, et al., JETP 88, 148 (1999).

[17] A.S. Alexandrov, et al., Phys. Rev. Lett. 76983 (1996).
[18] C. Capan, et al., Phys. Rev. Lett. 88, 056601 (2002).

[19] J. W. Loram, et al., J. Superconductivity, 7, 243 (1994); V. M. Krasnov et al., Phys. Rev. Lett. 84, 5860(2000).

[20] N.F. Mott and E.A. Davis, Electronic Processes in NonCrystalline Materials, 2nd edition (Clarendon Press, Oxford, 1979).

[21] J. Tateno, in Polarons and Bipolarons in High-T $T_{c} \mathrm{Su}$ perconductors and Related Materials, eds. E.K.H. Salje, A.S. Alexandrov and W.Y. Liang (Cambridge University Press, 1995), p. 309.

[22] J. Clayhold, Phys. Rev. B 54, 6103 (1996).

[23] J.B. Goodenough, J-S. Zhou, J. Chan, Phys. Rev. B47, 5275 (1993); J.R. Cooper et al., ibid 35, 8794 (1987); Y. Nakamura, S. Uchida, ibid 47, 8369 (1993); H.Y. Hwang et al., Phys. Rev. Lett. 72, 2636 (1994); Y. Fukuzumi et al., ibid 76, 684, (1996).

[24] J.E. Enderby, A.C. Barnes, Phys. Rev. B49, 5062 (1994).

[25] B.I. Shklovskii, Soviet Phys. JETP Lett. 36, 51 (1982).

[26] R. Mansfield, H. Tokumoto, Phil Mag. B48, L1 (1983).

[27] H. B ottger, V.V. Bryksin, Hopping Conduction in Solids (Verlag, Berlin, 1985) pp. 111, 283.

[28] The magnetic field $B \gtrsim 1$ Tesla is strong in conventional semiconductors with shallow impurity levels [26].

[29] D.Y. Xing, M. Liu, Phys. Rev. B43, 3744 (1991).

[30] A.S. Alexandrov, A.M. Bratkovsky, N.F. Mott, Phys. Rev. Lett. 72, 1734 (1994).

[31] A.S. Alexandrov, J. Low Temp. Phys. 87, 721 (1992).

[32] A.S. Alexandrov, Theory of Superconductivity ( IoP Publishing, Bristol and Philadelphia, 2003).

[33] T. Lopez-Arias, A. Smerzi, Phys. Rev. A 58, 526 (1998).

[34] V.N. Zavaritsky, V.V. Kabanov, A.S. Alexandrov, Europhys. Lett. 60, 127, (2002) and references therein. 